\title{
Mixed volume and distance geometry techniques for counting Euclidean embeddings of rigid graphs
}

\author{
Ioannis Z. Emiris ${ }^{1}$, Elias P. Tsigaridas ${ }^{2}$, and Antonios Varvitsiotis ${ }^{3}$ \\ 1 University of Athens, Greece, emiris@di.uoa.gr \\ 2 INRIA Paris-Rocquencourt, France, elias@polsys.lip6.fr \\ 3 Centrum Wiskunde \& Informatica (CWI), The Netherlands, A.Varvitsiotis@cwi.nl
}

Summary. A graph $G$ is called generically minimally rigid in $\mathbb{R}^{d}$ if, for any choice of sufficiently generic edge lengths, it can be embedded in $\mathbb{R}^{d}$ in a finite number of distinct ways, modulo rigid transformations. Here, we deal with the problem of determining tight bounds on the number of such embeddings, as a function of the number of vertices. The study of rigid graphs is motivated by numerous applications, mostly in robotics, bioinformatics, sensor networks and architecture. We capture embeddability by polynomial systems with suitable structure, so that their mixed volume, which bounds the number of common roots, yields interesting upper bounds on the number of embeddings. We explore different polynomial formulations so as to reduce the corresponding mixed volume, namely by introducing new variables that remove certain spurious roots, and by applying the theory of distance geometry. We focus on $\mathbb{R}^{2}$ and $\mathbb{R}^{3}$, where Laman graphs and 1-skeleta (or edge graphs) of convex simplicial polyhedra, respectively, admit inductive Henneberg constructions. Our implementation yields upper bounds for $n \leq 10$ in $\mathbb{R}^{2}$ and $\mathbb{R}^{3}$, which reduce the existing gaps and lead to tight bounds for $n \leq 7$ in both $\mathbb{R}^{2}$ and $\mathbb{R}^{3}$; in particular, we describe the recent settlement of the case of Laman graphs with 7 vertices. Our approach also yields a new upper bound for Laman graphs with 8 vertices, which is conjectured to be tight. We also establish the first lower bound in $\mathbb{R}^{3}$ of about $2.52^{n}$, where $n$ denotes the number of vertices.

Key words: rigid graph, Laman graph, Euclidean embedding, Henneberg construction, polynomial system, mixed volume, Cayley-Menger matrix, cyclohexane caterpillar

\subsection{Introduction}

Rigid graphs (or mechanisms) constitute an old but still very active area of research due to their deep mathematical and algorithmic questions, as well as numerous applications, notably in mechanism and linkage theory [10, 23, 44, 45, 48], structural bioinformatics [19, 35, 32, 42], sensor network localization [22, 33, 49], and architecture [21, 25].

We start this section by introducing notation and some necessary definitions. Throughout, $G=(V, E)$ denotes a simple loop-less graph with $|V|=n$. A framework of $G$ consists of an assignment of vectors $p_{1}, \ldots, p_{n} \in \mathbb{R}^{d}$ to the nodes of the graph, denoted by $G(p)$. Two frameworks $G(p)$ and $G(q)$ are called equivalent if $\left\|p_{i}-p_{j}\right\|=\left\|q_{i}-q_{j}\right\|, \forall(i, j) \in E$ and they are called congruent if $\left\|p_{i}-p_{j}\right\|=\left\|q_{i}-q_{j}\right\|, \forall i, j \in V$. A framework $G(p)$ is called rigid if there exists an $\epsilon>0$ such that if $\|p-q\|<\epsilon$ and $G(q)$ is equivalent to $G(p)$ then they are congruent. Equivalently, a framework $G(p) \in \mathbb{R}^{d}$ is called rigid if (the orbit of) $p$ is an isolated point in the orbit space of the group of Euclidean motions acting on the real variety $\mathbb{V}(p)=\left\{x \in \mathbb{R}^{d n}:\left\|x_{i}-x_{j}\right\|^{2}=\left\|p_{i}-p_{j}\right\|^{2}, \forall(i, j) \in E\right\}$. It is a well known fact that for real closed semi-algebraic sets the sum of the Betti numbers is finite, so in particular the number of connected components is finite [2]. This implies that for a given rigid framework $G(p)$ there exists a finite number of frameworks which are equivalent but not congruent to it.

A framework $G(p)$ is called generic if its coordinates are algebraically independent over $\mathbb{Q}$. A graph $G$ is called generically rigid if every generic framework of $G$ is rigid. Equivalently, a graph is generically rigid in $\mathbb{R}^{d}$ if, for generic edge lengths, it can be embedded in $\mathbb{R}^{d}$ in a finite number of ways, modulo congruence transformations. Here, a congruence transformation refers to either a translation or a rotation. A graph is minimally rigid if it is no longer rigid once any edge is removed. The problem of interest in this paper is to determine the maximum number of distinct planar and spatial Euclidean embeddings of generically minimally rigid, or simply rigid, graphs, up to rigid transformations, as a function of the number of vertices.

A graph $G=([n], E),[n]=\{1, \ldots, n\}$, is called Laman if $|E|=2 n-3$, and, additionally, all of its vertex-induced subgraphs with $3 \leq k<n$ vertices to have less than $2 k-3$ edges. This is related to the Chebychev-Grübler- 
Kutzbach's formula on the degrees of freedom for mechanical linkages, e.g. [1]. It is a fundamental theorem that the class of Laman graphs coincides with the generically minimally rigid graphs in $\mathbb{R}^{2}[34,38]$.

On the other hand, an analogous combinatorial characterization of rigidity in $\mathbb{R}^{3}$ has proven to be elusive and remains one of the most important open problems in the area of rigidity theory. In particular, the natural generalization of Laman's property in $\mathbb{R}^{3}$, i.e., $|E|=3 n-6$, and all vertex-induced subgraphs with $4 \leq k<n$ vertices have less than $3 k-6$ edges, is no longer sufficient to characterize minimal rigidity in 3 dimensions. The famous counterexample of the Double Banana (Figure 1.1) is a non-rigid graph in $\mathbb{R}^{3}$ which satisfies the necessary conditions mentioned above.

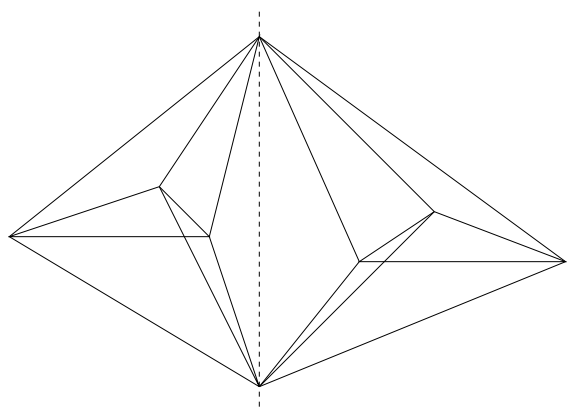

Fig. 1.1. The Double Banana; a non-rigid graph which satisfies the Laman property for $d=3$.

Thus we restrict our attention to graphs that correspond to the 1-skeleta, or edge graphs, of (convex) simplicial polyhedra; clearly, these graphs admit one convex embedding (modulo reflections as well). They are known to be generically minimally rigid in $\mathbb{R}^{3}[24]$.

Both Laman graphs and the 1-skeleta of simplicial polyhedra admit inductive constructions that begin with a simplex of the appropriate dimension, followed by a sequence of so-called Henneberg steps [47]. There are $d$ types of such steps in $\mathbb{R}^{d}$, each adding one new vertex and increasing the total number of edges by $d$, for $d=2,3$. They will be described in the sequel. A graph is Laman, or the 1-skeleton of a simplicial polytope, if it can be constructed by a sequence of the corresponding Henneberg steps.

To study upper bounds, we define a well-constrained polynomial system, that is polynomial systems with as many equations as unknowns, expressing the edge length constraints, whose real solutions correspond precisely to the different embeddings. When defining a straightforward system such as (1.1) and (1.6) in $\mathbb{R}^{2}$ and $\mathbb{R}^{3}$ respectively, all nontrivial equations are quadratic. There are $2 n-4$ and $3 n-9$ equations respectively, hence, by applying the classical Bézout bound on the number of common roots, we obtain $4^{n-2}$ and $8^{n-3}$. It is indicative of the hardness of the problem that efforts to substantially improve these bounds have failed $[6,41]$.

\subsubsection{Existing work}

The bound on the number of embeddings in dimension $d$ is

$$
\prod_{k=0}^{n-d-2} \frac{\left(\begin{array}{c}
n-1+k \\
n-d-1-k
\end{array}\right)}{\left(\begin{array}{c}
2 k+1 \\
k
\end{array}\right)}
$$

and is tight if we consider embeddings in $\mathbb{C}^{d}$. The bound was obtained by exploiting results from complex algebraic geometry that bound the degree of (symmetric) determinantal varieties defined by distance matrices [5, 6, 29]. For the planar and spatial case, it follows that the best known upper bounds are, respectively:

$$
\left(\begin{array}{c}
2 n-4 \\
n-2
\end{array}\right) \simeq 4^{n-2} / \sqrt{\pi(n-2)}, \text { and } \frac{2^{n-3}}{n-2}\left(\begin{array}{c}
2 n-6 \\
n-3
\end{array}\right) \simeq 8^{n-3} /((n-2) \sqrt{\pi(n-3)})
$$

In [41], mixed volumes (cf. Section 1.2) also yield an upper bound of $4^{n-2}$, for Laman graphs.

In applications, it is crucial to know the number of embeddings for specific (small) values of $n$. The most important result in this direction was to show that the Desargues graph admits precisely 24 embeddings in the plane [31, 26]. In different communities, this graph is known as the planar parallel robot, or the 3-prism graph. It is also known that the $K_{3,3}$ graph admits 16 embeddings in the plane [44], and the cyclohexane graph admits 16 embeddings in space (Section 1.5). 


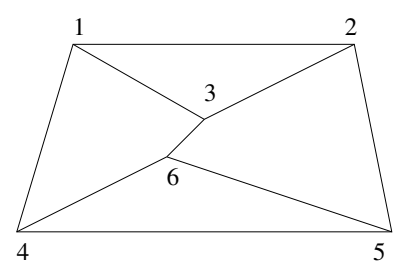

Fig. 1.2. The Desargues, or 3-prism, graph; equivalently, a planar parallel robot.

Another important question concerns lower bounds. For $\mathbb{R}^{2}$, there exist a lower bound of $24^{\lfloor(n-2) / 4\rfloor} \simeq 2.21^{n}$, obtained by a caterpillar construction, and one of $2 \cdot 12^{\lfloor(n-3) / 3\rfloor} \simeq 2.29^{n} / 6$, obtained by a fan ${ }^{4}$ construction [6]. Both bounds are based on the Desargues graph (Figure 1.2), which admits 24 embeddings. This bound has been slightly improved to $\Omega\left(2.3^{n}\right)$ [18], by using a construction which is based on the 7-vertex graph (Figure 1.5), which admits 56 embeddings. For the exact number of embeddings for certain rigid graphs, based on the Henneberg- 1 steps we refer the reader to [36].

\subsubsection{New results}

To upper bound the number of Euclidean embeddings of rigid graphs, we explore adequate polynomial systems leading to tight root bounds. This is a deep and hard question, with a wide range of applications in different fields. In the sequel, we shall shed some light to this issue. Our main tool is the mixed volume of a well-constrained polynomial system, which exploits the sparseness of the equations. This bounds the number of common roots, by Bernstein's Theorem 1, as described in Section 1.2. This bound is never larger than Bézout's, and is typically much tighter.

An alternative is to actually solve a system with coefficients chosen randomly and count the real roots, though this only yields an indication on the upper bound. The advantage of mixed volume is that it treats entire classes of systems defined by their nonzero terms, without considering specific coefficient values. In addition, a tight mixed volume implies that one can solve the system efficiently, either by sparse resultants [8], or by sparse homotopies [43]. More precisely, this means, respectively, that the sparse resultant matrix, or the number of homotopy paths, shall be (close to) optimal.

In the sequel, we derive the first lower bound in $\mathbb{R}^{3}$ :

$$
16^{\lfloor(n-3) / 3\rfloor} \simeq 2.52^{n}, n \geq 9
$$

by designing a cyclohexane caterpillar (Figure 1.10). Moreover, we have implemented specialized software that constructs all rigid graphs up to isomorphism, for small $n$, and computes the mixed volumes of the respective polynomial systems. We thus obtain upper and lower bounds for $n \leq 10$ in $\mathbb{R}^{2}$ and $\mathbb{R}^{3}$, which reduce the existing gaps, see Tables 1.1 and 1.2. Moreover, we establish tight bounds up to $n=7$ in $\mathbb{R}^{2}$ and $\mathbb{R}^{3}$ by appropriately reformulating the polynomial system. We describe in detail the case of 7-vertex Laman graphs, also known as 11-bar mechanisms in robotics, and establish a tight upper bound by distance geometry. We apply Bernstein's Second theorem (Theorem 2) to show that the naive polynomial system (1.6) cannot yield tight mixed volumes in the spatial case. Our approach also yields a new upper bound for Laman graphs with 8 vertices, which is conjectured to be tight. Our results indicate that mixed volume can be of general interest in enumeration problems.

The rest of the chapter is structured as follows. Section 1.2 presents our algebraic tools and our implementation, Section 1.3 discusses the planar case $(d=2)$, Section 1.4 outlines the theory of distance geometry and applies it to Laman graphs with $n=6,7$, Section 1.5 deals with $\mathbb{R}^{3}$, and we conclude with open questions and a conjecture. Several results appeared in [20] in preliminary form, whereas the tight count for 7-vertex Laman graphs was established in [18].

\subsection{Polynomial systems and mixed volume}

This section discusses multivariate polynomial systems, introduces mixed volume and describes our software.

Classical elimination theory characterizes every polynomial by its total degree. For a well-constrained system of polynomial equations, the classical Bézout bound on the number of isolated roots equals the product of the polynomials' total degrees. One disadvantage of this bound is that it counts complex projective roots and hence increases when there are roots at projective infinity.

\footnotetext{
${ }^{4}$ This corrects the exponent of the original statement.
} 
We introduce sparse elimination theory in order to exploit sparseness; for details, see [11]. In sparse (or toric) elimination theory, a polynomial is characterized by its support. Given a polynomial $f$ in $n$ variables, its support is the set of exponents in $\mathbb{N}^{n}$ corresponding to nonzero terms (or monomials). The Newton polytope of $f$ is the convex hull of its support and lies in $\mathbb{R}^{n}$. Consider polytopes $P_{i} \subset \mathbb{R}^{n}$ and parameters $\lambda_{i} \in \mathbb{R}, \lambda_{i} \geq 0$, for $i=1, \ldots, n$. We denote by $\lambda_{i} P_{i}$ the corresponding scalar multiple of $P_{i}$. Consider the Minkowski sum of the scaled polytopes $\lambda_{1} P_{1}+\cdots+\lambda_{n} P_{n} \in \mathbb{R}^{n}$; its $n$-dimensional (Euclidean) volume is a homogeneous polynomial of degree $n$ in the $\lambda_{i}$. The coefficient of the monomial $\lambda_{1} \cdots \lambda_{n}$ is the mixed volume of $P_{1}, \ldots, P_{n}$. If $P_{1}=\cdots=P_{n}$, then the mixed volume is $n$ ! times the volume of $P_{1}$. We focus on the topological torus $\mathbb{C}^{*}=\mathbb{C}-\{0\}$ in order to state the so-called BKK root bound, in terms of mixed volume.

Theorem 1. [3] Let $f_{1}=\cdots=f_{n}=0$ be a polynomial system in $n$ variables with real coefficients. The $f_{i}$ have fixed Newton polytopes, hence all coefficients corresponding to polytope vertices are nonzero. Then, the number of common isolated solutions in $\left(\mathbb{C}^{*}\right)^{n}$ is bounded above by the mixed volume of these Newton polytopes. This bound is tight for a generic choice of coefficients of the $f_{i}$ 's.

In fact, the theorem also holds for positive-dimensional zero-sets or, to be more precise, positive-dimensional toric varieties [11].

Bernstein's Second Theorem below describes genericity. Given $v \in\left(\mathbb{R}^{*}\right)^{n}$ and polynomial $f_{i}=\sum_{a \in A_{i}} c_{i, a} x^{a}$, we denote by $\partial_{v} f_{i}$ the polynomial obtained by keeping only those terms minimizing the inner product between their exponent vector and $v$. The Newton polytope of $\partial_{v} f_{i}$ is the face of the Newton polytope of $f_{i}$ supported by $v$.

Theorem 2. [3] If for all $v \in\left(\mathbb{R}^{*}\right)^{n}$ the face system $\partial_{v} f_{1}=\ldots=\partial_{v} f_{n}=0$ has no solutions in $\left(\mathbb{C}^{*}\right)^{n}$, then the mixed volume of the $f_{i}$ exactly equals the number of solutions in $\left(\mathbb{C}^{*}\right)^{n}$, and all solutions are isolated. Otherwise, the mixed volume is a strict upper bound on the number of isolated solutions.

This theorem was used to study planar embeddings [41]; we shall also apply it to $\mathbb{R}^{3}$.

We have developed specialized software that constructs all Laman graphs and all 1-skeleta of simplicial polyhedra in $\mathbb{R}^{3}$ with $n \leq 10$, using the respective Henneberg steps. Our computational platform is SAGE ${ }^{5}$, whereas Henneberg steps were implemented, using SAGE's interpreter, in Python. We classify all the graphs up to isomorhism using SAGE's interface for N.I.C.E., an open-source isomorphism check engine, keeping for each graph the Henneberg sequence with largest number of $H_{1}$ steps. For each graph we construct a polynomial system whose real solutions express all possible embeddings, using formulation (1.8) below. By genericity, solutions have no zero coordinates. For each system we bound the number of its complex solutions by computing its mixed volume. This can be computed by any relevant software. We used the implementation of the Lift-Prune algorithm in C [17], which can be called from within Maple ${ }^{6}$.

For every Laman graph, to discard translations and rotations, we pick an edge and fix the coordinates of its vertices, as shown in system (1.1). In $\mathbb{R}^{3}$, we choose a triangle and fix the coordinates of its vertices, as shown in (1.8). More generally, in $\mathbb{R}^{d}$, a $(d-1)$-dimensional simplex needs to be fixed. Depending on the choice of the fixed vertices, we obtain different systems hence different mixed volumes. Since they all bound the actual number of embeddings, we use the minimum mixed volume.

We used an Intel Core2, at $2.4 \mathrm{GHz}$, with $2 \mathrm{~GB}$ of RAM. We tested more that 20,000 graphs and computed the mixed volume of more than 40,000 polynomial systems. The total time of experiments was about 2 days. Tables 1.1 and 1.2 summarize our results.

\subsection{Laman graphs}

This section studies the number of embeddings of Laman graphs in $\mathbb{R}^{2}$. We discuss Henneberg steps, then study the number of embeddings for small $n$, and focus on $n=6$.

Let us introduce a family of simple systems, which has been used in the past, e.g. [41], to express embeddability in $\mathbb{R}^{2}$. Here $x_{i}, y_{i}$ denote the coordinates of the $i$-th vertex, and the $d_{i j}$ are the given lengths.

$$
\begin{cases}x_{i}=a_{i}, y_{i}=b_{i}, & i=1,2, \quad a_{i}, b_{i} \in \mathbb{R}, \\ \left(x_{i}-x_{j}\right)^{2}+\left(y_{i}-y_{j}\right)^{2}=d_{i j}^{2}, & (i, j) \in E-\{(1,2)\}\end{cases}
$$

The endpoints of edge $(1,2)$ are fixed, namely we fix $\left(x_{1}, y_{1}\right)$, e.g. to $(0,0)$, so as to remove translations, and $\left(x_{2}, y_{2}\right)$, e.g. to $(1,0)$ to remove rotations and scaling, assuming without loss of generality, that edge $(1,2)$ exists.

\footnotetext{
${ }^{5}$ http://www.sagemath.org/

${ }^{6}$ http://www.di.uoa.gr/ emiris/index-eng.html
} 
Let us consider the Henneberg steps defining Laman graphs, each adding a new vertex and a total number of two edges. A Henneberg-1 (or $H_{1}$ ) step connects the new vertex to two existing vertices (Figure 1.3). A Hennenerg-2 (or $\mathrm{H}_{2}$ ) step connects the new vertex to three existing vertices having at least one edge among them, and this edge is removed (Figure 1.4). We represent each Laman graph with $n \geq 3$ by $\triangle s_{4} \ldots, s_{n}$, where $s_{i} \in\{1,2\}$; this is known as its Henneberg sequence. The way to interpret this representation is that $G$ can be constructed inductively by starting with the simplex $\triangle$, where at step $i \geq 4$ a new vertex is inserted by performing a Henneberg step of type $s_{i} \in\{1,2\}$. Given a graph represented by sequence $S$, performing a $H_{1}$ step yields graph $S 1$, whereas performing a $H_{2}$ step yields graph $S 2$. The Desargues graph (Fig. 1.2) is represented by $\triangle 112$.

Note that this sequence is by no means unique for a given graph; moreover, the same sequence may yield different graphs. A Laman graph is called $H_{1}$ if it can be constructed using only $H_{1}$ steps; it is called $H_{2}$ otherwise.

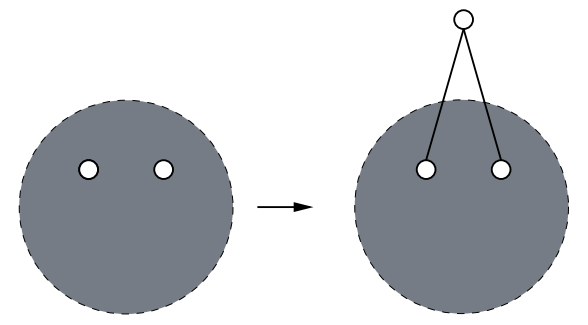

Fig. 1.3. A Henneberg-1 step (edge addition).

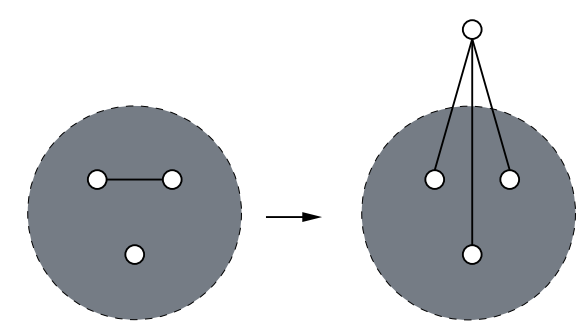

Fig. 1.4. A Henneberg-2 step (edge split).

Since two circles intersect generically in two points, a $H_{1}$ step at most doubles the number of embeddings and this is tight, generically. It follows that a $H_{1}$ graph with $n$ vertices has $2^{n-2}$ embeddings. One can easily verify that every $\triangle 2$ graph is isomorphic to a $\triangle 1$ graph and that every $\triangle 12$ graph is isomorphic to a $\triangle 11$ graph. Consequently, all Laman graphs with $n=4,5$ are $H_{1}$ and have 4 and 8 embeddings, respectively.

For a Laman graph with 6 vertices, a tight uppper bound of 24 follows by examining the three possibilities: the graph is either $H_{1}$, it is $K_{3,3}$, or is the Desargues graph. Now, $H_{1}$ graphs with 6 vertices have 16 embeddings. The $K_{3,3}$ graph has precisely 16 embeddings [44], a fact first conjectured in [48]. The Desargues graph has precisely 24 embeddings: the upper bound was first shown in [31] and proven more explicitly, along with the lower bound, in [26]. We return to the case $n=6$ below, and show how the Desargues bound is obtained as a mixed volume.

Table 1.1 summarizes our results for $n \leq 10$, including the result of Theorem 5 for $n=7$. Recently, the same approach has given a better upper bound of 116 for $n=8$, which is conjectured to be tight [13]. Using our software (Section 1.2), we construct all Laman graphs with $n=9,10$, and compute their respective mixed volumes, thus obtaining the shown upper bounds. The lower bound for $n=9$ follows from the Desargues fan [6], while the others follow from the fact that a $H_{1}$ step exactly doubles the number of embeddings.

\begin{tabular}{c||c|c|c|c||c|c|c|c}
$n=$ & 3 & 4 & 5 & 6 & 7 & 8 & 9 & 10 \\
\hline \hline upper & 2 & 4 & 8 & 24 & 56 & 116 & 512 & 2048 \\
\hline \hline lower & 2 & 4 & 8 & 24 & 56 & 112 & 288 & 576 \\
\hline \hline$H_{1}$ & $\triangle$ & $\triangle 1$ & $\triangle 11$ & $\triangle 111$ & $\triangle 1^{4}$ & $\triangle 1^{5}$ & $\triangle 1^{6}$ & \\
\hline \multirow{2}{*}{$H_{2}$} & & & & $\triangle \mathbf{1 1 2}$ & $\triangle \mathbf{1}^{3} \mathbf{2}$ & $\triangle \mathbf{1}^{4} \mathbf{2}$ & $\triangle 1^{5} 2$ & \\
& & & & & & $\triangle 1^{4} 21$ & \\
& & & & & & $\triangle \mathbf{1}^{\mathbf{4} 22}$ & \\
\hline
\end{tabular}

Table 1.1. Bounds and Henneberg sequences for Laman graphs for $n \leq 10$. Exponents indicate the number of times a certain Henneberg step is repeated. Bold text indicates the simplest Henneberg sequence yielding the upper bound.

We now establish a general upper bound, which improves upon the existing ones when our graph contains many degree-2 vertices.

Lemma 1. Let $G$ be a Laman graph with $n>6$ vertices among which there are $k$ degree-2 vertices. Then, the number of planar embeddings of $G$ is bounded above by $3\left(2^{k+2} 4^{n-k-6}\right)$.

Proof. Our proof parallels that of [41] which uses mixed volumes to bound the effect of a $H_{1}$ step, when it is the last one in the Henneberg sequence. We start by removing all of the $k$ degree- 2 vertices. Notice that the removal 
of a degree-2 vertex does not destroy other degree-2 vertices (because the remaining graph should be also Laman), although it may create new ones. The remaining graph has $n-k$ vertices, and according to Table 1.1 , the first 6 of them can only contribute 24 to the total number of embeddings.

\subsubsection{Algebraic formulations for $n=6$}

Let us now focus on $n=6$ and study good algebraic representations of the given problem, so that mixed volume offers interesting bounds. For the Desargues graph, an optimal mixed volume of 24 is obtained by using the general approach of distance geometry (see next section). Interestingly, we were not able to construct a polynomial system for $K_{3,3}$ whose mixed volume were optimal. Our best result is a system defined by distance matrices, yielding a bound of 25 .

Let us concentrate on planar quaternions, employed in [10] to derive a degree-6 resultant in $q_{4}$. Quaternions are widely used to provide rational parameterizations of rigid transformations. We define a planar quaternion:

$$
q:=\left[\frac{1}{2}\left(d_{x} \cos \frac{\theta}{2}+d_{y} \sin \frac{\theta}{2}\right), \frac{1}{2}\left(d_{y} \cos \frac{\theta}{2}-d_{x} \sin \frac{\theta}{2}\right), \sin \frac{\theta}{2}, \cos \frac{\theta}{2}\right] \in \mathbb{R}^{4},
$$

to express rotation by $\theta$ and translation by $\left(d_{x}, d_{y}\right)$ in the plane, where $q_{3}^{2}+q_{4}^{2}=1$. The corresponding transformation matrix is

$$
T:=\left[\begin{array}{ccc}
q_{4}^{2}-q_{3}{ }^{2} & -2 q_{3} q_{4} & 2 q_{1} q_{4}-2 q_{2} q_{3} \\
2 q_{3} q_{4} & q_{4}{ }^{2}-q_{3}{ }^{2} & 2 q_{1} q_{3}+2 q_{2} q_{4} \\
0 & 0 & 1
\end{array}\right]
$$

and is used to define the reference frame of one triangle with respect to that of the other triangle, by writing equations for the 3 edges linking the triangles, cf. Figure 1.2. The rest of edge lengths concern points within a reference frame and can be decoupled; their effect is to multiply the number of solutions by 4 .

The 3 equations above refer to edges $(i, i+3)$ for $i=1,2,3$; together with $q_{3}^{2}+q_{4}^{2}=1$, they define a well-constrained system. Let us take a closer look: they are all of a similar form, the simplest one being $\left|\left\langle T v_{1}, v_{4}\right\rangle\right|=d_{14}^{2}$, where $\langle\cdot, \cdot\rangle$ denotes inner product and $v_{1}, v_{4}$ are the origins of the two reference frames. The observation of [10] is that these 3 equations are homogeneous in the $q_{i}$ 's except from the $d_{i j}$ terms. It suffices then to multiply the latter by $q_{3}^{2}+q_{4}^{2}$ to obtain 3 homogeneous equations in new variables $z_{i}=q_{i} / q_{4}, i=1,2,3$. Now, the problem is reduced to a system of 3 non-homogeneous equations in $z_{1}, z_{2}, z_{3}$; its mixed volume is 6 . The fourth equation becomes $z_{3}^{2}+1=z_{0}$, which uniquely specifies $z_{0}=1 / q_{4}$ for each of the system's solutions. Hence, the overall number of embeddings is 24 , and this is optimal.

\subsection{Distance geometry}

In this section we introduce distance geometry and Cayley-Menger matrices, then combine results from distance geometry with mixed volume, which settled the case $n=7$ in [18]. Distance matrices were introduced by A. Cayley in 1841 with the aim to derive necessary conditions on the pairwise distances of 5 points in Euclidean space [9]. The theory behind distance geometry has been well developed, e.g. [4, 12, 16, 40]. Applications of distance geometry to structural bioinformatics, e.g. [30, 42], have been quite succesful in practice, e.g. [28, 37]. We refer to [14] and further references therein for a detailed study of Euclidean distance matrices.

\subsubsection{Preliminaries}

We begin by introducing some basic notation and necessary definitions. Let $\mathbb{S}_{n}$ denote the set of symmetric $n \times n$ matrices and $\mathbb{S}_{+}^{n}$ the set of $n \times n$ positive semidefinite matrices. Throughout, $e$ will denote the all ones vector of the appropriate size. Consider a matrix $D \in \mathbb{S}_{n}$ such that $D_{i j}=d_{i j}^{2}$ for every $i \neq j$ and $D_{i i}=0$ for $i \in[n]$. Such a matrix is usually called a pre-distance matrix (in other fields it is known as a dissimilarity matrix). A Euclidean distance matrix (EDM) is a pre-distance matrix $D$ for which there exist $p_{1}, \ldots, p_{n} \in \mathbb{R}^{k}$ such that

$$
D_{i j}=\left\|p_{i}-p_{j}\right\|^{2}, 1 \leq i, j \leq n,
$$

where $\|\cdot\|$ denotes Euclidean distance. Let $\operatorname{EDM}_{n}$ denote the cone of $n \times n$ Euclidean distance matrices. Vectors $p_{1}, \ldots, p_{n}$ realize the pre-distance matrix $D$ if they satisfy relation (1.2). Given $D \in \operatorname{EDM}_{n}$, let ed $(D)$ denote the smallest dimension $k$ in which there exist vectors realizing $D$. This is called the embedding dimension of $D$.

The previous definitions lead to some natural questions. Can we identify necessary and sufficient conditions that will ensure that a given pre-distance matrix is a EDM? Moreover, given $D \in \mathrm{EDM}_{n}$, can we compute its embedding dimension? Theorem 3 below, provides positive answers to both questions. 
Theorem 3. $[27,40]$ Let $D \in \mathbb{S}_{n}$ be a pre-distance matrix and vector $s \in \mathbb{R}^{n}$ such that $s^{T} e=1$. Then, $D \in \mathrm{EDM}_{\mathrm{n}}$ if and only if

$$
F_{s}(D):=-\frac{1}{2}\left(I-e s^{T}\right) D\left(I-s e^{T}\right) \text { is positive semidefinite. }
$$

Moreover, if $D \in \mathrm{EDM}_{n}$ then $\operatorname{ed}(D)=\operatorname{rank} F_{s}(D)$.

Theorem 3 implies that testing embeddabililty of a pre-distance matrix $D$ can be done in polynomial time, since it amounts to checking whether some matrix is positive semidefinite. Additionally, the embedding dimension can also be efficiently computed, since it amounts to a rank computation. We now obtain a useful reformulation of Theorem 3 by Cayley-Menger determinants. The Cayley-Menger matrix associated with a given pre-distance matrix $D \in \mathbb{S}_{n}$ is the $(n+1) \times(n+1)$ matrix

$$
\mathrm{CM}(D)=\left(\begin{array}{cc}
0 & e \\
e^{T} & D
\end{array}\right) .
$$

It is an easy exercise to check that the Schur complement of $\operatorname{CM}(D)$ with respect to the $2 \times 2$ submatrix $\left(\begin{array}{ll}0 & 1 \\ 1 & 0\end{array}\right)$, indexed by the first row/column and the $i$-th row/column is equal to $-2 F_{e_{i}}(D)$. Consequently,

$$
\begin{aligned}
\operatorname{det} \operatorname{CM}(D) & =(-1)^{n} 2^{n-1} \operatorname{det} F_{e_{i}}(D), \\
\operatorname{rankCM}(D) & =\operatorname{rank} F_{e_{i}}(D)+2 .
\end{aligned}
$$

Now, we express the condition of $F_{e_{i}}(D)$ being positive semidefinite in terms of Cayley-Menger determinants. It is well known that $F_{e_{i}}(D) \in \mathbb{S}_{n}^{+}$if and only if every principal minor of $F_{e_{i}}(D)$ has non-negative determinant. But the principal minors of $F_{e_{i}}(D)$ have the form $F_{e_{i}}(D[X])$, for some $X \subseteq[n]$, where $D[X]$ denotes the principal minor of $D$ indexed by $X$. This observation, combined with (1.3), forms the crux of the following reformulation of Theorem 3 .

Theorem 4. [39] Let $D \in \mathbb{S}_{n}$ be a pre-distance matrix. Then $D \in \mathrm{EDM}_{\mathrm{n}}$ if and only if

$$
\forall X \subseteq[n], \quad(-1)^{|X|} \operatorname{det} \mathrm{CM}(D[X]) \geq 0 .
$$

Condition (1.4) yields inequalities. For $|X|=2$, it expresses the fact that all entries of $D$ must be non-negative. For $|X|=3$, it captures the triangular inequality. Indeed, if we apply it to $X=\{1,2,3\}$, then condition (1.4) becomes:

$$
\left(d_{12}+d_{13}+d_{23}\right)\left(d_{12}+d_{13}-d_{23}\right)\left(d_{12}+d_{23}-d_{13}\right)\left(d_{13}+d_{23}-d_{12}\right) \leq 0,
$$

with equality satisfied precisely when the corresponding points are collinear, and strict inequality satisfied when the points define a triangle. Equivalently, for $X=\{i, j, k\}$ condition (1.4) can be written as $d_{i k}+d_{j k} \geq d_{i j}$ for all triplets $i, j, k \in\{1, \ldots, n\}$. For $k=4$ the condition captures the tetrangular inequality.

We are now ready to summarize our approach. Consider a Laman graph $G=([n], E)$, together with an assignment of generic weights $d_{i j} \in \mathbb{R}_{+}$to its edges. Let $D$ be the corresponding partial (symmetric) matrix, i.e., $D_{i j}=d_{i j}^{2}, \forall(i, j) \in E$ and $\operatorname{diag}(D)=0$. A matrix $D^{\prime} \in \mathbb{S}_{n}$ is called an EDM completion of $D$, if $D^{\prime} \in \mathrm{EDM}_{n}$ and $D_{i j}^{\prime}=D_{i j}, \forall(i, j) \in E$. Clearly, the number of embeddings of the distances $d=\left(d_{i j}\right)$ in the plane, modulo rigid transformations, is equal to the number of EDM completions of $D$ that satisfy $\operatorname{rankCM}(D) \leq 4$.

The condition $\operatorname{rankCM}(D)=4$ imposes the vanishing of all $5 \times 5$ minors of $\mathrm{CM}(D)$. Thus, we obtain a polynomial system of $\left(\begin{array}{c}n+1 \\ 5\end{array}\right)$ equations in the $\left(\begin{array}{c}n \\ 2\end{array}\right)-2 n+3$ unknowns $x_{i j}$, corresponding to the lengths of the edges that are not present in $G$. Clearly, the number of its real solutions is an upper bound on the number of embeddings of $G$ in $\mathbb{R}^{2}$. We employ mixed volumes in order to bound the number of complex solutions.

For this, we identify all square subsystems which correspond to Laman subgraphs of $G$ and compute their mixed volume. Focusing on Laman subgraphs implies that we make use of Cayley-Menger minors only, in other words our system is defined by submatrices whose first row and column are filled with ones. This is indispensable for the system to have a finite number of solutions. We thus construct several well-constrained polynomial systems, each yielding a mixed volume, the smallest of which provides the desired upper bound.

\subsubsection{The case $n=6$}

Consider the Desargues graph and its associated Cayley-Menger matrix seen below. Here $c_{i j}=d_{i j}^{2}$ correspond to the fixed distances, and $x_{15}, x_{16}, x_{24}, x_{26}, x_{34}, x_{35}$ to the unspecified ones. The numbering of the vertices corresponds to that in Figure 1.2. Moreover, we assume that the matrix is indexed by $0,1, \ldots, 6$ and we use the shorthand notation $\mathrm{CM}(X)$ for the principal minor defined by the indices in $X$. 


$v_{1}$
$v_{2}$
$v_{3}$
$v_{4}$
$v_{5}$
$v_{6}$$\left[\begin{array}{ccccccc}0 & 1 & 1 & 1 & 1 & 1 & 1 \\ 1 & 0 & c_{12} & c_{13} & c_{14} & x_{15} & x_{16} \\ 1 & c_{12} & 0 & c_{23} & x_{24} & c_{25} & x_{26} \\ 1 & c_{13} & c_{23} & 0 & x_{34} & x_{35} & c_{36} \\ 1 & c_{14} & x_{24} & x_{34} & 0 & c_{45} & c_{46} \\ 1 & x_{15} & c_{25} & x_{35} & c_{45} & 0 & c_{56} \\ 1 & x_{16} & x_{26} & c_{36} & c_{46} & c_{56} & 0\end{array}\right]$

By Theorem 4 or conditions (1.3), all $5 \times 5$ principal minors of this matrix that contain the first row/column of ones vanish, hence yielding polynomial equations on the variables $x_{15}, x_{16}, x_{24}, x_{26}, x_{34}, x_{35}$. There are $\left(\begin{array}{c}6 \\ 4\end{array}\right)=15$ such minors, 9 of which yield bivariate and the rest trivariate equations. We restrict attention to bivariate equations, which turn out to be sufficient in this case. There are 6 quadratics and 3 cubics. No $3 \times 3$ subsystem exists, but it is possible to find several $4 \times 4$ subsystems, including some with only one cubic. Take, for example, the minors

$$
\mathrm{CM}(0,2,4,5,6)\left(x_{24}, x_{26}\right)=\operatorname{CM}(0,1,4,5,6)\left(x_{15}, x_{16}\right)=\operatorname{CM}(0,1,2,4,5)\left(x_{15}, x_{24}\right)=\operatorname{CM}(0,1,2,3,6)\left(x_{16}, x_{26}\right)=0
$$

where we have indicated the variables per equation. They define a system of 3 quadratic and one cubic equation in $x_{15}, x_{16}, x_{24}, x_{26}$, and its mixed volume is 24 . The other two unknowns are uniquely defined from each solution because one can easily construct linear equations expressing each of the $x_{34}, x_{35}$, in terms of the $x_{15}, x_{16}, x_{24}, x_{26}$. For instance $x_{16} \mathrm{CM}(0,1,2,3,4)-\mathrm{CM}(0,1,3,4,6)$ is linear in $x_{34}$ and quadratic in $x_{16}, x_{24}$.

Let us examine $K_{33}$ with the same approach. We obtain the following Cayley-Menger matrix, where $c_{i j}=d_{i j}^{2}$ correspond to the fixed distances for $i=1,3,5, j=2,4,6$, and $x_{13}, x_{15}, x_{24}, x_{26}, x_{35}, x_{46}$ are the unspecified distances.

\begin{tabular}{|c|c|c|c|c|c|c|}
\hline & $-\begin{array}{ll} & v_{1} \\
0 & 1\end{array}$ & $\begin{array}{c}v_{2} \\
1\end{array}$ & $\begin{array}{r}v_{3} \\
1\end{array}$ & $\begin{array}{r}v_{4} \\
1\end{array}$ & $\begin{array}{c}v_{5} \\
1\end{array}$ & $\begin{array}{c}v_{6} \\
1\end{array}$ \\
\hline$v_{1}$ & 10 & $c_{12}$ & $x_{13}$ & $c_{14}$ & ${ }_{1} x_{15}$ & $c_{16}$ \\
\hline$v_{2}$ & $1 c_{12}$ & 0 & $c_{23}$ & $x_{24}$ & ${ }_{4} c_{25}$ & $x_{26}$ \\
\hline$v_{3}$ & $1 x_{13}$ & $c_{23}$ & 0 & $c_{34}$ & ${ }_{4} x_{35}$ & $c_{36}$ \\
\hline$v_{4}$ & $1 c_{14}$ & $x_{24}$ & $c_{34}$ & 0 & $c_{45}$ & $x_{46}$ \\
\hline$v_{5}$ & $1 x_{15}$ & $c_{25}$ & $x_{35}$ & $c_{45}$ & $5 \quad 0$ & $c_{56}$ \\
\hline & $1 c_{16}$ & $x_{26}$ & $c_{36}$ & $x_{46}$ & $6 c_{56}$ & 0 \\
\hline
\end{tabular}

We consider the $5 \times 5$ minors that yield cubic bivariate polynomials in $x_{13}, x_{15}, x_{24}, x_{26}, x_{35}, x_{46}$. No $3 \times 3$ subsystem exists, but it is possible to find several $4 \times 4$ subsystems, such as the following:

$$
\mathrm{CM}(0,3,4,5,6)\left(x_{35}, x_{46}\right)=\mathrm{CM}(0,2,3,5,6)\left(x_{26}, x_{35}\right)=\mathrm{CM}(0,1,4,5,6)\left(x_{15}, x_{46}\right)=\mathrm{CM}(0,1,2,5,6)\left(x_{15}, x_{26}\right)=0 .
$$

This system has a mixed volume of 25 . Furthermore, the above equations are sparse enough to reveal precise information about their common solutions via univariate (i.e., Sylvester) resultants. Specifically, we can consider the first two and the last two polynomials, respectively, as univariate in $x_{35}$ and $x_{15}$, and obtain resultants $R_{1}$ and $R_{2}$ in $x_{26}, x_{46}$. Seen as univariate polynomials in $x_{26}$, they yield a resultant in $x_{46}$ that factorizes into the product of $x_{46}^{2}$, a factor of degree 16, and another of degree 8; analyzing these factors may yield an upper bound of 16.

\subsubsection{The case $n=7$}

Let us consider Laman graphs with 7 vertices. We will prove the main result of this section, namely a tight count of embeddings, which was established in [18].

If a $H_{1}$ step is applied to any graph with $n=6$, the resulting graph with $n=7$ admits exactly 48 embeddings. To maximize the number of embeddings, we shall apply a $H_{2}$ step to any graph with $n=6$. By checking graph isomorphisms and taking into account the various symmetries, it was shown [20] that there are only 3 relevant graphs to be considered. These are obtained by a $\mathrm{H}_{2}$ step applied to the Desargues graph as follows, where numbering refers to Figure 1.2: We remove edge $(4,5)$, and add edges $(4,7),(5,7)$ and one of the following 3 edges: $(1,7)$, $(3,7)$, or $(6,7)$. The first case corresponds to the topology that shall be studied extensively in the sequel, since it leads to the maximum number of 56 embeddings. We will call this graph $G_{7}$ and it is shown in Figure 1.5. The other two graphs admit at most 44 and 48 embeddings, respectively, hence they are not studied any further. These upper bounds are obtained as mixed volumes of polynomial systems, as explained above.

Theorem 5. [18] The maximum number of planar Euclidean embeddings for a Laman graph with 7 vertices is 56. 


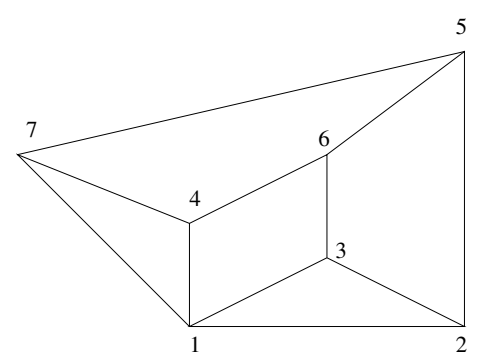

Fig. 1.5. The graph that gives the tight bound for $n=7$.

Proof. It is known that for the graph of Figure 1.5, there exist edge lengths for which it has 56 embeddings in $\mathbb{R}^{2}$ [18]. This settles the lower bound; the rest of the proof focuses on the upper bound.

The Cayley-Menger matrix for graph $G_{7}$ is below, where the $c_{i j}=d_{i j}^{2}$ correspond to the fixed distances, and $x_{i j}$ are the unspecified distances.

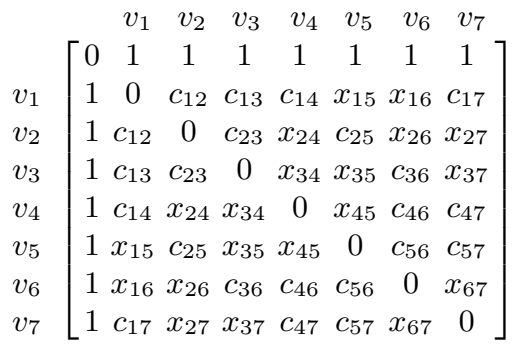

By Theorem 4, any $5 \times 5$ minor of this matrix must vanish, which yields polynomial equations on the variables $x_{i j}$. There are $\left(\begin{array}{l}8 \\ 5\end{array}\right)$ such minors, but only $\left(\begin{array}{l}7 \\ 4\end{array}\right)=35$ Cayley-Menger minors, each in 2 to 4 variables. Among these polynomials, no $4 \times 4$ subsystem exists that corresponds to a rigid mechanism, as was verified by checking Laman's condition in our Maple implementation.

However, it is possible to find certain $5 \times 5$ subsystems whose subgraph is Laman and, moreover, uniquely define the configuration of the overall graph. One of these systems has 4 bivariate equations and one trivariate equation, and is defined by taking the following minors:

$$
\left\{\begin{array}{l}
\operatorname{CM}(0,4,5,6,7)\left(c_{46}, c_{47}, c_{56}, c_{57}, x_{45}, x_{67}\right)=0 \\
\operatorname{CM}(0,1,4,6,7)\left(c_{14}, c_{17}, c_{46}, c_{47}, x_{16}, x_{67}\right)=0 \\
\operatorname{CM}(0,1,4,5,7)\left(c_{14}, c_{17}, c_{47}, c_{57}, x_{15}, x_{45}\right)=0 \\
\operatorname{CM}(0,1,2,3,5)\left(c_{12}, c_{13}, c_{25}, c_{23}, x_{15}, x_{35}\right)=0 \\
\operatorname{CM}(0,1,3,5,6)\left(c_{13}, c_{36}, c_{56}, x_{15}, x_{16}, x_{35}\right)=0
\end{array}\right.
$$

These define a system of 3 quadratic and two cubic equations in $x_{15}, x_{16}, x_{35}, x_{45}, x_{67}$; the cubics are the first and last polynomials. The corresponding subgraph is Laman and, moreover, once the unknown lengths are fixed, they uniquely define the configuration of the overall graph. The reason is that, once we solve for the unknowns in the system, the resulting graph has more constraints than given by Laman's condition.

The mixed volume turns out to be equal to 56. This bounds the number of complex common solutions of the system, hence the number of real embeddings. Notice that this bound does not take into account solutions with zero coordinates, in other words some zero length. However, a graph has embeddings with some zero length only when the input bar lengths form a singular set, in the sense that they would satisfy a non-generic algebraic dependency. For example, by letting some input distance be exactly 0, some graph may theoretically have infinitely many configurations. However, generically, it is impossible to have such an embedding. Thus for $n=7$ we have a tight bound of 56 .

\subsection{1-skeleta of simplicial polyhedra}

This section extends the previous results to 1-skeleta, or edge-skeleta, of (convex) simplicial polyhedra, which are known to be rigid in $\mathbb{R}^{3}$.

Fewer results are known on the number of embeddings of such graphs, despite their relevance in applications. One related work in robotics studies all classes of parallel robots [23] but focuses on the generic number of complex 
configurations. Concerning real configurations, the most celebrated result states that the general Stewart (or Gough) platform has 40 real positions [15].

To express embeddability in $\mathbb{R}^{3}$, we extend system (1.1), where $x_{i}, y_{i}, z_{i}$ denote the coordinates of the $i$-th vertex, and the $d_{i j}$ are the given lengths:

$$
\begin{cases}x_{i}=a_{i}, y_{i}=b_{i}, z_{i}=c_{i}, & i=1,2,3, \quad a_{i}, b_{i}, c_{i} \in \mathbb{R} \\ \left(x_{i}-x_{j}\right)^{2}+\left(y_{i}-y_{j}\right)^{2}+\left(z_{i}-z_{j}\right)^{2}=d_{i j}^{2}, & (i, j) \in E-\{(1,2),(1,3),(2,3)\}\end{cases}
$$

We fix $\left(x_{1}, y_{1}, z_{1}\right)=(0,0,0)$ to remove translations, $\left(x_{2}, y_{2}, z_{2}\right)=(1,0,0)$ to remove 2 rotational degrees of freedom and scaling, and set $x_{3}=0, z_{3}>0$ to remove the third rotational degree of freedom. If we choose 3 points forming a triangle, then we can compute the unique position of $\left(x_{3}, y_{3}, z_{3}\right)$ as in system (1.6).

Consider any $k+2$ vertices forming a cycle with $\geq k-1$ diagonals, $k \geq 1$. The (extended) Henneberg- $k$ step (or $\left.H_{k}\right), k=1,2,3$, corresponds to adding a vertex, connecting it to the $k+2$ vertices, and removing $k-1$ diagonals among them, as illustrated in Figure 1.6. Since 3 spheres intersect generically in two points, a $H_{1}$ step at most doubles the number of spatial embeddings and this is tight, generically.

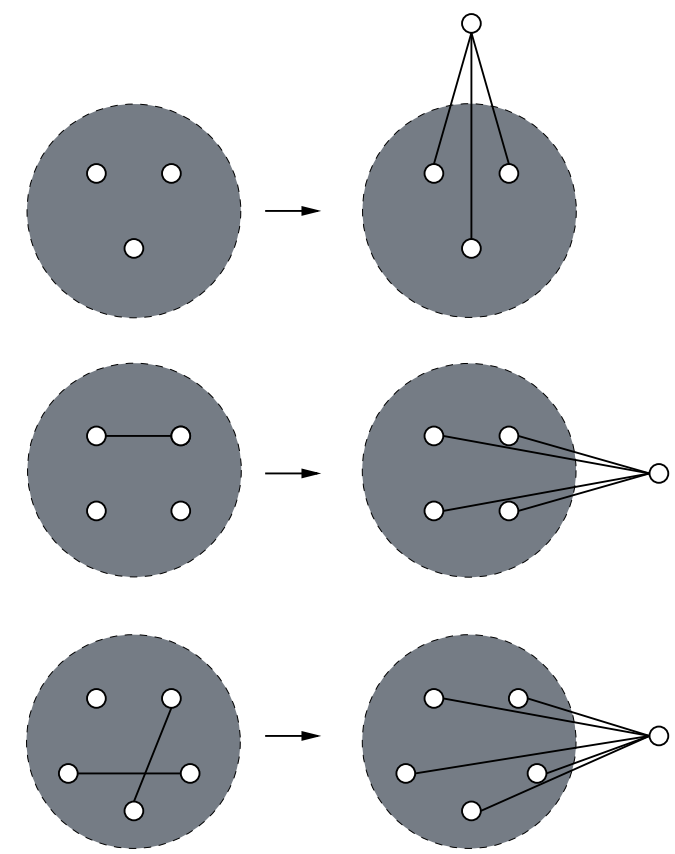

Fig. 1.6. The spatial Henneberg steps.

Proposition 1. [7] A graph is the 1-skeleton of a simplicial polyhedron in $\mathbb{R}^{3}$ if and only if it has a construction that begins with a tetrahedron, followed by any sequence of $\mathrm{H}_{1}, \mathrm{H}_{2}, \mathrm{H}_{3}$ steps.

System (1.6) has $3 n$ unknowns. Our first observation is that this system does not capture the structure of the problem. Specifically, by choosing direction

$$
v=(\underbrace{0,0,0}_{v_{1}}, \underbrace{0,0,0}_{v_{2}}, \underbrace{0,0,0}_{v_{3}}, \underbrace{-1,-1-1}_{v_{4}}, \ldots, \underbrace{-1,-1,-1}_{v_{n}}) \in \mathbb{R}^{3 n}
$$

the corresponding face system becomes:

$$
\begin{cases}x_{i}=a_{i}, y_{i}=b_{i}, z_{i}=c_{i}, & i=1,2,3, \quad a_{i}, b_{i}, c_{i} \in \mathbb{R}, \\ x_{i}^{2}+y_{i}^{2}+z_{i}^{2}=0, & (i, j) \in E: i \notin\{1,2,3\}, j \in\{1,2,3\}, \\ \left(x_{i}-x_{j}\right)^{2}+\left(y_{i}-y_{j}\right)^{2}+\left(z_{i}-z_{j}\right)^{2}=0, & (i, j) \in E, i, j \notin\{1,2,3\} .\end{cases}
$$

This system has $\left(a_{1}, b_{1}, c_{1}, \ldots, a_{3}, b_{3}, c_{3}, 1,1, \gamma \sqrt{2}, \ldots, 1,1, \gamma \sqrt{2}\right) \in\left(\mathbb{C}^{*}\right)^{3 n}$ as a solution, where $\gamma= \pm \sqrt{-1}$. Consequently, according to Theorem 2, the mixed volume of system (1.6) is not a tight bound on the number of solutions 
in $\left(\mathbb{C}^{*}\right)^{3 n}$. To remove spurious solutions (at toric infinity), we introduce variables $w_{i}=x_{i}^{2}+y_{i}^{2}+z_{i}^{2}$, for $i=1, \ldots, n$. This yields the following equivalent system, but with lower mixed volume:

$$
\begin{cases}x_{i}=a_{i}, y_{i}=b_{i}, z_{i}=c_{i}, & i=1,2,3 \\ w_{i}=x_{i}^{2}+y_{i}^{2}+z_{i}^{2}, & i=4, \ldots, n \\ w_{i}+w_{j}-2 x_{i} x_{j}-2 y_{i} y_{j}-2 z_{i} z_{j}=d_{i j}^{2}, & (i, j) \in E-\{(1,2),(1,3),(2,3)\} .\end{cases}
$$

This is the formulation we have used in our computations. The reduced mixed volume is indicated by a face system similar to the one previously defined. To be more specific, we consider $v \in \mathbb{R}^{4 n-3}$ such that $v_{i}=0$ for $i \leq 9$ and $i>3 n$, where the latter coordinates correspond to $w_{4}, \ldots, w_{n}$, and $v_{i}=-1$ for $i=10, \ldots, 3 n$. The corresponding face system becomes:

$$
\begin{cases}x_{i}=a_{i}, y_{i}=b_{i}, z_{i}=c_{i}, & i=1,2,3, \quad a_{i}, b_{i}, c_{i} \in \mathbb{R}, \\ x_{i}^{2}+y_{i}^{2}+z_{i}^{2}=0, & i \in\{4, \ldots, n\} \\ x_{i} x_{j}+y_{i} y_{j}+z_{i} z_{j}=0, & (i, j) \in E, i, j \notin\{1,2,3\} \\ x_{i} x_{j}+y_{i} y_{j}+z_{i} z_{j}=0, & (i, j) \in E: i \notin\{1,2,3\}, j \in\{1,2,3\},\end{cases}
$$

where the first two classes of equations are similar to the corresponding ones in system (1.7). In particular, this face system does not admit roots like those above, with $\left(x_{i}, y_{i}, z_{i}\right)=(1,1, \gamma \sqrt{2})$ for $i=4, \ldots, n$, because of the fourth set of equations. Of course, there are probably other face systems with nontrivial roots.

We continue by establishing tight bounds for some small cases. For $n=4$, the only simplicial polytope is the 3 -simplex, which clearly admits only 2 embeddings. For $n=5$, there is a unique graph that corresponds to a 1-skeleton of a simplicial polyhedron [7], cf. Figure 1.7. This graph is obtained from the 3-simplex through a $H_{1}$ step, so for $n=5$ there is a tight bound of 4 .

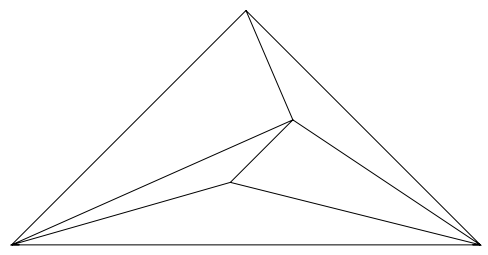

Fig. 1.7. The only 1-skeleton of a simplicial polytope on 5 vertices.

Lemma 2. The 1-skeleton of a simplicial polyhedron on 6 vertices has at most 16 embeddings, and this bound is tight.
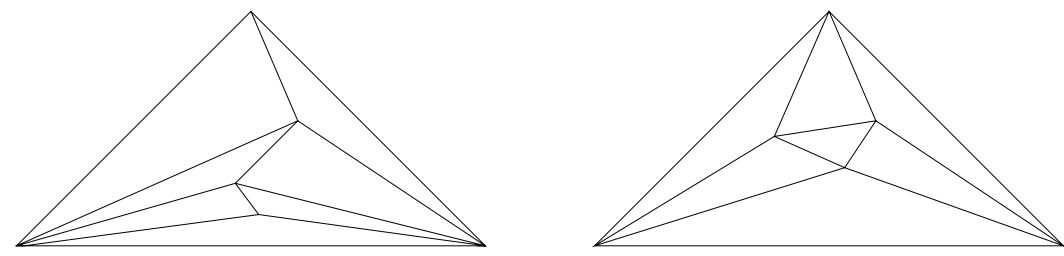

Fig. 1.8. All 1-skeleta of convex simplicial polyhedra on 6 vertices.

Proof. There are two non-isomorphic graphs $G_{1}, G_{2}$ for $n=6$ [7], cf. Figure 1.8. For $G_{1}$, the mixed volume is 8 . Since all facets of $G_{2}$ are symmetric, we fix one and compute the mixed volume, which equals 16 , so the overall upper bound is 16 . We shall obtain a matching lower bound.

Notice that $G_{2}$ is the graph of the cyclohexane, which admits 16 distinct Euclidean embeddings for generic edge lengths [19]. To see the equivalence, recall that the cyclohexane is essentially a 6-cycle (cf. Figure 1.9), with known lengths between vertices at distance 1 (adjacent) and 2 .

The former are bond lengths whereas the latter are specified by the angle between consecutive bonds. In [19], the upper bound was obtained as the mixed volume of two different systems; one was composed of equations obtained by distance matrices. 

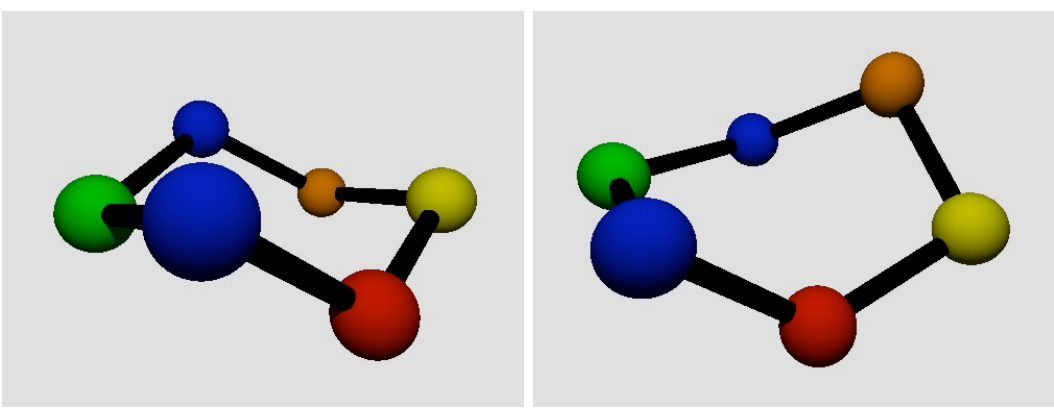

Fig. 1.9. A chair and a boat configuration of the cyclohexane molecule.

Alternatively, $G_{2}$ corresponds to a Stewart platform parallel robot with 16 configurations, where two triangles define the platform and base, and 6 lengths link the triangles in a jigsaw shape. A folklore argument gives the configurations by placing two points per axis ${ }^{7}$. On each axis, one point belongs to the platform, the other to the base. There are two choices for labeling points on each axis, giving 8 configurations; by reflection about the origin, we obtain another 8 .

We now pass to general $n$ and establish the first lower bound in $\mathbb{R}^{3}$.

Theorem 6. There exist edge lengths for which the cyclohexane caterpillar construction has $16^{\lfloor(n-3) / 3\rfloor} \simeq 2.52^{n}$ embeddings, for $n \geq 9$.

Proof. We glue together copies of cyclohexanes sharing a common triangle. The resulting graph is the 1-skeleton of a simplicial polytope. Each new copy adds 3 vertices, and since there exist edge lengths for which the cyclohexane graph has 16 embeddings the claim follows.

A caterpillar made of two cyclohexane copies is illustrated in Figure 1.10.

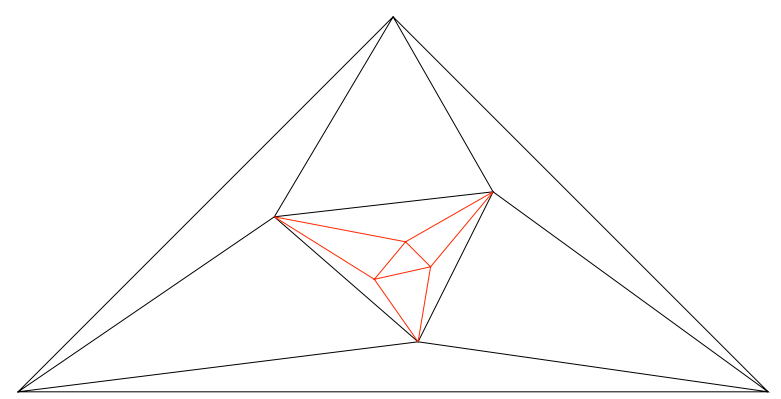

Fig. 1.10. A cyclohexane caterpillar with 2 copies.

Table 1.2 summarizes our computational results for $n \leq 10$, where $\triangle$ is the 3 -simplex, and bold text indicates the Henneberg sequence of the graph that yields the upper bound. The upper bounds for $n=7, \ldots, 10$ are computed by our software employing mixed volumes. The lower bound for $n=9$ follows from Theorem 6 . All other lower bounds are obtained by applying a $H_{1}$ step to a graph with one fewer vertex.

Our computation also shows that, in constructing all non-isomorphic 1-skeleta of simplicial polyhedra, $H_{3}$ need not be applied before $n=13$. Lastly, we state a result similar to Lemma 1.

Lemma 3. Let $G$ be the 1-skeleton of a simplicial polyhedron on $n>7$ vertices among which there are $k$ degree-3 vertices. Then the number of embeddings of $G$ is bounded above by $2^{k+5} 8^{n-k-7}$.

Proof. We start by removing all of the $k$ degree- 3 vertices. Notice that the removal of a degree- 3 vertex does not destroy other degree-3 vertices (because the remaining graph should be also the edge graph of a simplicial polyhedron), although it may create new ones. The remaining graph has $n-k$ vertices, and according to Table 1.2 , the first 7 of them can only contribute 32 to the total number of embeddings.

\footnotetext{
${ }^{7}$ Personal communication with Daniel Lazard.
} 


\begin{tabular}{c||c|c||c|c|c|c|c}
$n=$ & 4 & 5 & 6 & 7 & 8 & 9 & 10 \\
\hline \hline upper & 2 & 4 & 16 & 32 & 160 & 640 & 2560 \\
\hline \hline lower & 2 & 4 & 16 & 32 & 64 & 256 & 512 \\
\hline \hline$H_{1}$ & $\triangle$ & $\triangle 1$ & $\triangle 11$ & $\triangle 111$ & $\triangle 1^{4}$ & $\triangle 1^{5}$ & $\triangle 1^{6}$ \\
\hline & & & $\triangle \mathbf{1 2}$ & $\triangle \mathbf{1}^{\mathbf{2}} \mathbf{2}$ & $\triangle 1^{3} 2$ & $\triangle 1^{4} 2$ & $\triangle 1^{5} 2$ \\
$H_{2}$ & & & & & $\triangle \mathbf{1}^{\mathbf{2}} \mathbf{2}^{\mathbf{2}}$ & $\triangle 1^{3} 2^{2}$ & $\triangle 1^{4} 2^{2}$ \\
& & & & $\triangle 1^{2} 21$ & $\triangle 1^{3} 21$ & $\triangle 1^{4} 21$ \\
& & & & & $\triangle \mathbf{1}^{\mathbf{2}} \mathbf{2}^{\mathbf{3}}$ & $\triangle 1^{3} 2^{3}$ \\
& & & & & & $\triangle 1^{3} 21^{2}$ \\
& & & & & & $\triangle 1^{3} 212$ \\
& & & & & & $\triangle 1^{3} 2^{2} 1$ \\
\hline$H_{3}$ & & & & & & & \\
\hline
\end{tabular}

Table 1.2. Bounds and Henneberg sequences for 1 -skeleta of simplicial polyhedra for $n \leq 10$, where $\triangle$ is the 3 -simplex, and bold are the Henneberg sequences yielding the upper bound.

\subsection{Further work}

Undoubtedly, the most important and oldest problem in rigidity theory is the full combinatorial characterization of rigid graphs in $\mathbb{R}^{3}$. In the planar case, existing bounds are not tight. This is due to the fact that root counts include rotated copies of certain embeddings. Our approach based on distance matrices may offer an algorithmic process for obtaining good algebraic representations, in particular low mixed volumes, including in the spatial case. For high $n$ the issue is that the number of equations produced is quite large, with algebraic dependancies among them.

Since we deal with Henneberg constructions, it is important to determine the effect of each step on the number of embeddings: a $H_{1}$ step always doubles their number; we conjecture that $H_{2}$ multiplies it by $\leq 4$ and spatial $H_{3}$ by $\leq 8$, but these may not always be tight. Our conjecture has been verified for small $n$.

The structures studied here are called point-and-bar structures; they generalize to body-and-bar, where edges can be connected to different points of a rigid body, and to body-and-hinges structures, where the allowed degrees of freedom model hinges, useful in structural bioinformatics. It is known that a body-and-bar structure in $\mathbb{R}^{d}$ is rigid if and only if the associated graph is the edge-disjoint union of $\left(\begin{array}{c}d+1 \\ 2\end{array}\right)$ spanning trees [47]. A similar result holds for body-and-hinges structures, where every hinge corresponds to $\left(\begin{array}{c}d+1 \\ 2\end{array}\right)-1$ edges [46].

Acknowledgements. I.Z. Emiris is partially supported by FP7 contract PITN-GA-2008-214584 SAGA: Shapes, Algebra, and Geometry. Part of this work was done while he was on sabbatical at team Salsa of INRIA Rocquencourt. E. Tsigaridas is partially supported by an individual postdoctoral grant from the Danish Agency for Science, Technology and Innovation, and also acknowledges support from the Danish National Research Foundation and the National Science Foundation of China (under grant 61061130540) for the Sino-Danish Center for the Theory of Interactive Computation, within which part of this work was performed, and from the EXACTA grant of the National Science Foundation of China (NSFC 60911130369) and the French National Research Agency (ANR-09-BLAN-037101). E. Tsigaridas performed part of this work while he was with the Aarhus University, Denmark. A. Varvitsiotis started work on this project as a graduate student at the University of Athens.

\section{References}

1. J. Angeles. Rational Kinematics. Springer-Verlag, New York, 1989.

2. S. Basu, R. Pollack, and M-F.Roy. Algorithms in Real Algebraic Geometry, volume 10 of Algorithms and Computation in Mathematics. Springer-Verlag, 2nd edition, 2006.

3. D.N. Bernstein. The number of roots of a system of equations. Funct. Anal. and Appl., 9(2):183-185, 1975.

4. L.M. Blumenthal. Theory and Applications of Distance Geometry, volume 15. Chelsea Publishing Company, Bronx, NY, 2nd edition, 1970.

5. C. Borcea. Point configurations and Cayley-Menger varieties, 2002. arXiv:math/0207110.

6. C. Borcea and I. Streinu. The number of embeddings of minimally rigid graphs. Discrete Comp. Geometry, 31(2):287-303, 2004.

7. R. Bowen and S. Fisk. Generation of triangulations of the sphere. Mathematics of Computation, 21(98):250-252, 1967. 
8. J.F. Canny and I.Z. Emiris. A subdivision-based algorithm for the sparse resultant. J. ACM, 47(3):417-451, May 2000.

9. A. Cayley. On a theorem in the geometry of position. Cambridge Mathematical Journal, 2:267-271, 1841.

10. C.L. Collins. Forward kinematics of planar parallel manipulators in the Clifford algebra of $P^{2}$. Mechanism E Machine Theory, 37(8):799-813, 2002.

11. D. Cox, J. Little, and D. O'Shea. Using Algebraic Geometry. Number 185 in GTM. Springer, New York, 2nd edition, 2005.

12. J. Dattorro. Convex Optimization \& Euclidean Distance Geometry. Meboo USA, 2011.

13. S.C. Despotakis and I.Z. Emiris. An upper bound on Euclidean embeddings of rigid graphs with 8 vertices. Technical report, arxiv.org, 2012. http://arxiv.org/abs/1204.6527.

14. M.M. Deza and M. Laurent. Geometry of cuts and metrics. Springer, 1997.

15. P. Dietmeier. The Stewart-Gough platform of general geometry can have 40 real postures. In J. Lenarcic and M. Husty, editors, Advances in Robot Kinematics: Analysis and Control, pages 7-16. Springer-Verlag, New York, 1998.

16. A.W.M. Dress and T.F. Havel. Distance geometry and geometric algebra. Foundations of Physics, 23(10):1357$1374,1991$.

17. I.Z. Emiris and J.F. Canny. Efficient incremental algorithms for the sparse resultant and the mixed volume. J. Symbolic Computation, 20(2):117-149, 1995.

18. I.Z. Emiris and G. Moroz. The assembly modes of rigid 11-bar linkages. In Proc. IFToMM World Congress in Mechanism \& Machine Science, Guanajuato, Mexico, 2011.

19. I.Z. Emiris and B. Mourrain. Computer algebra methods for studying and computing molecular conformations. Algorithmica, Special Issue on Algorithms for Computational Biology, 25:372-402, 1999.

20. I.Z. Emiris, E. Tsigaridas, and A. Varvitsiotis. Algebraic methods for counting Euclidean embeddings of rigid graphs. In Graph Drawing, LNCS, pages 195-200. Springer, 2009.

21. D.G. Emmerich. Structures Tendues et Autotendantes. Ecole d'Architecture de Paris la Villette, 1988.

22. T. Eren, D.K. Goldenberg, W. Whiteley, Y.R. Yang, A.S. Morse, B.D.O. Anderson, and P.N. Belhumeur. Rigidity, computation and randomization in network localization. In Proc. IEEE INFOCOM'04, Hong Kong, pages 26732684, 2004.

23. J.C. Faugère and D. Lazard. The combinatorial classes of parallel manipulators combinatorial classes of parallel manipulators. Mechanism \& Machine Theory, 30(6):765-776, 1995.

24. H. Gluck. Almost all simply connected closed surfaces are rigid. Lect. Notes in Math., 438:225-240, 1975.

25. V. Gomez-Jauregui. Tensegrity Structures and their Application to Architecture. Servicio de Publicaciones, Universidad de Cantabria, Santander, Spain, 2010. Also: MSc Thesis, School of Architecture, Queen's University, Belfast, 2004.

26. C.M. Gosselin, J. Sefrioui, and M.J. Richard. Solutions polynomiales au problème de la cinématique directe des manipulateurs parallèles plans à trois degrés de liberté. Mechanism and Machine Theory, 27(2):107-119, 1992.

27. J.C. Gower. Euclidean distance geometry. Math. Sci, 1:1-14, 1982.

28. P. Guentert, C. Mumenthaler, and K. Wuthrich. Torsion angle dynamics for NMR structure calculation with the new program Dyana. J. Mol. Bio., 273:283-298, 1997.

29. J. Harris and L.W. Tu. On symmetric and skew-symmetric determinantal varieties. Topology, 23(1):71-84, 1984.

30. T.F. Havel. Distance geometry: Theory, algorithms, and chemical applications. In P. von Ragué, P. R. Schreiner, N. L. Allinger, T. Clark, J. Gasteiger, P. A. Kollman, and H. F. Schaefer III, editors, Encyclopedia of Computational Chemistry, pages 723-742. J. Wiley \& Sons, 1998.

31. K.N. Hunt. Structural kinematics of in parallel actuated robot arms. Trans. ASME, J. Mech. Transm. Autom. Des., pages 705-712, 1983.

32. D.J. Jacobs, A.J. Rader, L.A. Kuhn, and M.F. Thorpe. Protein flexibility predictions using graph theory. Proteins: Structure, Function, and Genetics, 44(2):150-165, 2001.

33. N. Krislock and H. Wolkowicz. Explicit sensor network localization using semidefinite representations and facial reductions. SIAM J. Optimization, 20(5):2679-2708, 2010.

34. G. Laman. On graphs and rigidity of plane skeletal structures. Journal of Engineering Mathematics, 4:331-340, October 1970.

35. C. Lavor, A. Mucherino, L. Liberti, and N. Maculan. On the computation of protein backbones by using artificial backbones of hydrogens. J. of Global Optimization, 50(2):329-344, 2011.

36. L. Liberti, C. Lavor, B. Masson, and A. Mucherino. Polynomial cases of the discretizable molecular distance geometry problem. Arxiv preprint arXiv:1103.1264, 2011.

37. T. Malliavin and F. Dardel. Structure des protéines par RMN. In Sciences Fondamentales, volume AF, pages 6608 (1-18). Techniques de l'Ingénieur, Paris, January 2002.

38. J.C. Maxwell. On the calculation of the equilibrium and stiffness of frames. Philosophical Magazine, 39(12), 1864.

39. K. Menger. Géométrie générale. In Mémorial des Sciences Mathématiques, volume 124. 1954. 
40. I.J. Schönberg. Remarks to M. Frechet's article "Sur la définition axiomatique d'une classe d'espaces vectoriels distanciés applicables vectoriellement sur l'espace de Hilbert". Annals of Math., 36:724-732, 1935.

41. R. Steffens and T. Theobald. Mixed volume techniques for embeddings of laman graphs. Computational Geometry: Theory and Applications, 43(2):84-93, 2010.

42. M.F. Thorpe and P.M. Duxbury, editors. Rigidity theory and applications. Fund. Materials Res. Ser. Kluwer, 1999.

43. J. Verschelde. Algorithm 795: PHCpack: a general-purpose solver for polynomial systems by homotopy continuation. ACM Trans. Math. Softw., 25(2):251-276, 1999.

44. D. Walter and M. Husty. On a 9-bar linkage, its possible configurations and conditions for paradoxical mobility. In Proc. IFToMM World Congress in Mechanism \& Machine Science, Besançon, France, 2007.

45. D. Walter and M.L. Husty. A spatial 9-bar linkage, possible configurations and conditions for paradoxical mobility. In Proc. NaCoMM, pages 195-208, Bangalore, India, 2007.

46. W. Whiteley. Rigidity and scene analysis. In J.E. Goodman and J. O'Rourke, editors, Handbook of Discrete and Computational Geometry, chapter 60, pages 893-916. CRC Press, Boca Raton, Florida, 2nd edition, 2004.

47. W. Whiteley and T.S. Tay. Generating isostatic frameworks. Structural topology, 11:21-69, 1985.

48. W. Wunderlich. Gefärlice annahmen der trilateration und bewegliche afchwerke I. Zeitschrift für Angewandte Mathematik und Mechanik, 57:297-304, 1977.

49. Z. Zhu, A. M.-C. So, and Y. Ye. Universal rigidity and edge sparsification for sensor network localization. SIAM J. Optimization, 20(6):3059-3081, October 2010. 